This item was submitted to Loughborough's Research Repository by the author.

Items in Figshare are protected by copyright, with all rights reserved, unless otherwise indicated.

\title{
Enhanced gel formation in binary mixtures of nanocolloids with short-range attraction
}

PLEASE CITE THE PUBLISHED VERSION

https://doi.org/10.1063/1.5007038

\section{PUBLISHER}

(c) American Institute of Physics

\section{VERSION}

VoR (Version of Record)

\section{PUBLISHER STATEMENT}

This work is made available according to the conditions of the Creative Commons Attribution-NonCommercialNoDerivatives 4.0 International (CC BY-NC-ND 4.0) licence. Full details of this licence are available at: https://creativecommons.org/licenses/by-nc-nd/4.0/

\section{LICENCE}

CC BY-NC-ND 4.0

\section{REPOSITORY RECORD}

Harden, James L., Hongyu Guo, Martine Bertrand, Tyler Shendruk, Subramanian Ramakrishnan, and Robert L. Leheny. 2019. "Enhanced Gel Formation in Binary Mixtures of Nanocolloids with Short-range Attraction". figshare. https://hdl.handle.net/2134/36370. 


\section{Enhanced gel formation in binary mixtures of nanocolloids with short-range attraction}

James L. Harden, Hongyu Guo, Martine Bertrand, Tyler N. Shendruk, Subramanian Ramakrishnan, and Robert L. Leheny

Citation: The Journal of Chemical Physics 148, 044902 (2018);

View online: https://doi.org/10.1063/1.5007038

View Table of Contents: http://aip.scitation.org/toc/jcp/148/4

Published by the American Institute of Physics

\section{Articles you may be interested in}

Vitrification and gelation in sticky spheres

The Journal of Chemical Physics 148, 044501 (2018); 10.1063/1.5000263

Communication: Modeling electrolyte mixtures with concentration dependent dielectric permittivity

The Journal of Chemical Physics 148, 041102 (2018); 10.1063/1.5018195

An atomistic fingerprint algorithm for learning ab initio molecular force fields

The Journal of Chemical Physics 148, 034101 (2018); 10.1063/1.5008630

Simulating structure and dynamics in small droplets of 1-ethyl-3-methylimidazolium acetate

The Journal of Chemical Physics 148, 193802 (2018); 10.1063/1.5010342

Perspective: Surface freezing in water: A nexus of experiments and simulations

The Journal of Chemical Physics 147, 060901 (2017); 10.1063/1.4985879

Quantum theory of multiscale coarse-graining

The Journal of Chemical Physics 148, 102335 (2018); 10.1063/1.5010270

\section{AIP| The Journal of Chemical Physics}




\title{
Enhanced gel formation in binary mixtures of nanocolloids with short-range attraction
}

\author{
James L. Harden, ${ }^{1}$ Hongyu Guo, ${ }^{2}$ Martine Bertrand, ${ }^{1}$ Tyler N. Shendruk, ${ }^{3}$ \\ Subramanian Ramakrishnan, ${ }^{4}$ and Robert L. Leheny ${ }^{2}$ \\ ${ }^{1}$ Department of Physics, University of Ottawa, Ottawa, Ontario K1N 6N5, Canada \\ ${ }^{2}$ Department of Physics and Astronomy, Johns Hopkins University, Baltimore, Maryland 21218, USA \\ ${ }^{3}$ Center for Studies in Physics and Biology, The Rockefeller University, 1230 York Avenue, New York, \\ New York 10065, USA \\ ${ }^{4}$ Department of Chemical and Biomedical Engineering, FAMU-FSU College of Engineering, Tallahassee, \\ Florida 32312, USA
}

(Received 29 September 2017; accepted 14 December 2017; published online 25 January 2018)

\begin{abstract}
Colloidal suspensions transform between fluid and disordered solid states as parameters such as the colloid volume fraction and the strength and nature of the colloidal interactions are varied. Seemingly subtle changes in the characteristics of the colloids can markedly alter the mechanical rigidity and flow behavior of these soft composite materials. This sensitivity creates both a scientific challenge and an opportunity for designing suspensions for specific applications. In this paper, we report a novel mechanism of gel formation in mixtures of weakly attractive nanocolloids with modest size ratio. Employing a combination of x-ray photon correlation spectroscopy, rheometry, and molecular dynamics simulations, we find that gels are stable at remarkably weaker attraction in mixtures with size ratio near two than in the corresponding monodisperse suspensions. In contrast with depletion-driven gelation at larger size ratio, gel formation in the mixtures is triggered by microphase demixing of the species into dense regions of immobile smaller colloids surrounded by clusters of mobile larger colloids that is not predicted by mean-field thermodynamic considerations. These results point to a new route for tailoring nanostructured colloidal solids through judicious combination of interparticle interaction and size distribution. Published by AIP Publishing. https://doi.org/10.1063/1.5007038
\end{abstract}

\section{INTRODUCTION}

The formation of colloidal gels is a ubiquitous process in nature, ${ }^{1,2}$ the control of which is crucial to a diverse range of technologies, including those related to the ceramics, food processing, and pharmaceuticals industries. The phase behavior and rheological properties of colloidal suspensions further make them a fertile ground for investigating fundamental issues in soft materials. In particular, as colloidal suspensions are among the simplest and most accessible systems for the study of ergodic to non-ergodic transformations, they have served as important models for addressing theoretical questions regarding glass and gel transitions. Mixing different colloidal species greatly expands the possible structure-property relationships realized by colloidal suspensions. From paints and advanced ceramics ${ }^{3-5}$ to the protein combinations that ensure clarity of the human eye lens, ${ }^{6}$ mixtures provide essential control of the microstructure and rheology of suspensions. Recent work on gel formation focusing on mixtures with different inter-species interactions has observed interesting contrasts with the properties of the gels formed by single-species suspensions. ${ }^{7-9}$ Another avenue for creating mixtures is by varying the size distribution of colloids, such as by introducing a binary mixture of sizes. However, research investigating the role of such size distributions as a parameter in gel formation has been limited. ${ }^{10-12}$
Most attention on polydisperse colloidal suspensions has focused on binary mixtures in which the intrinsic colloidal interactions are essentially hard-sphere-like. In this case, entropically driven depletion effects that create short-range attraction between the larger colloids represent the dominant interaction. The implications of this attraction have been studied in detail in theory, simulation, and experiment. ${ }^{13-24}$ For example, when the size ratio of the colloids, $K=R_{L} / R_{S}$, where $R_{L}$ and $R_{S}$ are the radii of the larger and smaller particles, respectively, is sufficiently large, $\kappa \geq 5$, these entropic effects ${ }^{14}$ make suspensions unstable with a number of possible consequences, including spinodal decomposition and the formation of coexisting fluid phases, ${ }^{16,18,20}$ crystallization of the larger colloids, ${ }^{13,15,16,22}$ and gel formation by the larger colloids due to arrested phase separation. ${ }^{1,21,23,24}$ To the extent that intrinsic interactions between the colloids (e.g., residual screened Coulombic repulsion, van der Waals attraction, or chemically specific surface interactions) in such binary mixtures have been considered, they have typically been treated as perturbations that modify the depletion-dominated phase behavior. ${ }^{1,25-36}$

In this paper, we explore a complementary perspective in which the colloids have a tunable intrinsic short-range attraction that can drive a fluid-gel transition even in monodisperse suspensions and ask how the additional influence of a modest binary size disparity modifies the suspension phase behavior, microstructural dynamics, and rheology. ${ }^{10,11}$ Studying such 
mixtures with a combination of rheometry, $\mathrm{x}$-ray photon correlation spectroscopy (XPCS), and molecular dynamics simulations, we obtain a picture connecting the microscopic behavior of the suspensions and their macroscopic mechanical response. We unexpectedly find that the gel formation does not follow a simple mixing law but instead is markedly enhanced in the mixtures over that of the corresponding monodisperse suspensions. Notably, the microscopic signature of the gelation is the dynamic arrest of the smaller species, in contrast to the expectations from thermodynamic calculations or depletioninduced gelation considerations. This observation opens a new strategy employing size disparity and colloidal interactions in tandem to control rheology and phase behavior of colloidal suspensions.

\section{RESULTS}

The experiments employed octadecyl-coated silica nanocolloids in decalin. ${ }^{37-40}$ Decalin is a marginal solvent for the hydrocarbon chains so that at high temperature, the chains form a brush corona on the particle surfaces that leads effectively to a hard repulsion and stabilizes the suspensions. ${ }^{40}$ (See Appendix A for details regarding sample preparation and other experimental procedures.) When the temperature is decreased below the theta temperature, $T_{\theta} \approx 307 \mathrm{~K}$, the octadecyl chains prefer to interpenetrate, creating a short-range attractive well between the colloids, extending $\approx 1 \mathrm{~nm}$ from the surface, with a depth $U(T)$ that increases roughly linearly with decreasing temperature, $U(T)=-A k_{B}\left(T-T_{\theta}\right)$, where $A \approx 30 . .^{38,41}$ As a consequence of this attraction, when the suspensions are cooled to a sufficiently low temperature, they undergo a transformation from a fluid state with a viscosity matching that expected for a suspension of hard spheres to a disordered gel state with solid-like mechanical properties. ${ }^{40}$ A number of studies have characterized various properties of this transformation in monodisperse suspensions including the phase boundary as a function of colloid volume fraction, the structure of the fluid and gel states, and the linear and nonlinear rheology of the gels. ${ }^{37-42}$ Recent work by our group and others has further investigated the temporal evolution of the rheology and particle-scale dynamics, using a combination of rheometry and XPCS, to identify quantitative relationships between the macroscopic mechanical behavior and microscopic motions in the gels. ${ }^{43-45}$

Here, we use this set of tools in conjunction with simulations to explore gel formation in suspensions of bidisperse mixtures of these colloids. The mixtures contained colloids of radii $R_{L} \approx 16 \mathrm{~nm}$ and $R_{S} \approx 8 \mathrm{~nm}$ (i.e., $\kappa \approx 2$ ), with a size polydispersity of approximately $10 \%$. Two series of mixtures were studied. In each series, the sum of the small-colloid volume fraction $\phi_{S}$ and large-colloid volume fraction $\phi_{L}$ was fixed at $\phi_{T}=\phi_{S}+\phi_{L}$, and the relative concentration of the smaller colloids was parameterized by $c=\phi_{S} / \phi_{T}$. In one series, $\phi_{T}=0.45$ and in the other, $\phi_{T}=0.30$.

To characterize the fluid-gel transition in the suspensions, we performed measurements of the complex shear modulus, $G^{*}(\omega)=G^{\prime}(\omega)+i G^{\prime \prime}(\omega)$. Figure 1(a) shows the storage modulus $G^{\prime}(\omega)$ and dissipation factor, $D(\omega)=G^{\prime \prime}(\omega) / G^{\prime}(\omega)$, at $\omega=1 \mathrm{~s}^{-1}$ for three suspensions with $\phi_{T}=0.45$ on heating at a
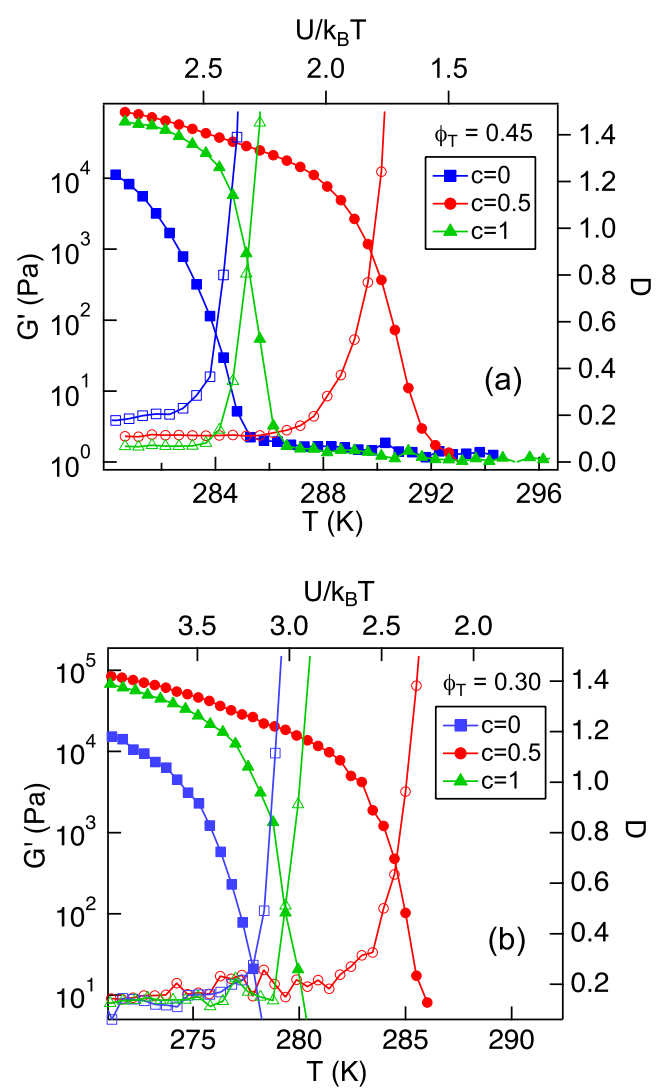

FIG. 1. Storage modulus $G^{\prime}$ (solid symbols) and dissipation factor $D$ (open symbols) at $\omega=1 \mathrm{~s}^{-1}$ of suspensions of mixtures of octadecyl silica colloids in decalin with radii of $8 \mathrm{~nm}$ and $16 \mathrm{~nm}(\kappa=2)$ as a function of temperature (or equivalently, as a function of the magnitude of the attractive well depth $U$ ) on heating at $0.1 \mathrm{~K} / \mathrm{min}$. The sum of the total volume fraction of colloids was fixed at (a) $\phi_{T}=0.45$ or (b) $\phi_{T}=0.30$, and results are shown at different relative concentration, $c=\phi_{S} / \phi_{T}$, of the smaller colloids, $c=0$ (blue squares), $c=0.5$ (red circles), and $c=1$ (green triangles).

ramp rate of $0.1 \mathrm{~K} / \mathrm{min}$ from low temperatures where the suspensions are gels to high temperatures where the short-range attraction vanishes and the suspensions become fluid. In each case, the modulus decreases precipitously and the dissipation factor increases, marking a gel-fluid transition whose position in temperature is insensitive to heating rate over a large range $(0.05-1 \mathrm{~K} / \mathrm{min})$. We identify the melting point of the gels with the temperature $T^{*}$ at which $D=1 .{ }^{43}$ Consistent with previous studies, ${ }^{37,39}$ the gel transition in monodisperse suspensions of the smaller colloids, $T^{*}(c=1) \approx 285.5 \mathrm{~K}$, is slightly higher than that of suspensions of the larger colloids, $T^{*}(c=0)$ $\approx 284 \mathrm{~K}$. Remarkably, for the symmetric mixtures, $c=0.5$, $T^{*}$ does not interpolate between these transition temperatures but is significantly higher, $T^{*}(c=0.5) \approx 290 \mathrm{~K}$. Figure $1(\mathrm{~b})$ shows results of equivalent measurements at $\phi_{T}=0.30$, which display the same trend except with $T^{*}$ at all $c$ shifted lower, as expected for suspensions with lower overall concentration. ${ }^{39}$ We note that this non-monotonic trend is robust against the precise definition of the gel temperature. For example, rheology studies of the gelation time of octadecyl-silica suspensions following rapid cooling have shown that the time required for gelation appears to diverge continuously for increasingly shallow quenches, and this divergence point has been identified as an alternative definition of the gel temperature. ${ }^{42}$ 


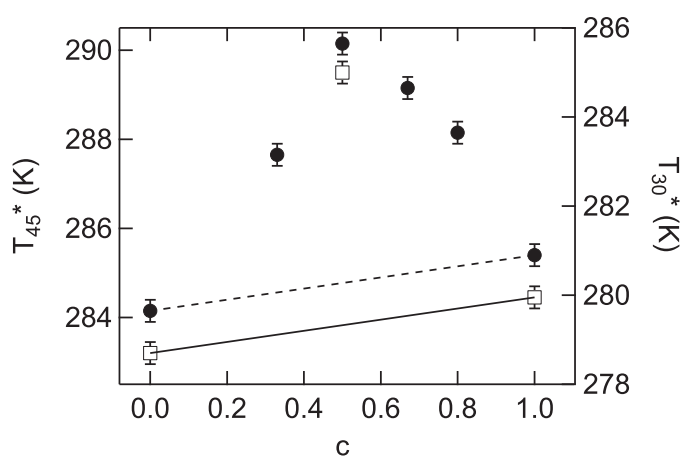

FIG. 2. Gel transition temperature as a function of the fraction $c$ of small colloids. Solid circles are for mixtures with $\phi_{T}=0.45$, and open squares are for mixtures with $\phi_{T}=0.30$. The dashed (solid) line between $c=0$ and 1 indicates the putative behavior for ideal mixing in the case of $\phi_{T}=0.45$ $\left(\phi_{T}=0.30\right)$.

Measurements on the suspensions with $\phi_{T}=0.45$ indicate that this divergence temperatures at $c=0.5$ lies several degrees above the corresponding temperature in the monodisperse suspensions, consistent with the trends in Fig. 2.

We stress that the modest size asymmetry of the mixtures, $\kappa \approx 2$, is far below that expected to cause phase separation due to depletion effects, and calculations for hard-sphere mixtures at these total concentrations and size ratio indicate well-mixed binary liquids..$^{46,47}$ Consistent with the absence of large-scale phase separation, the samples are visibly homogeneous and optically translucent in both the fluid and gel phases. Figure 2 shows $T^{*}$ for several values of $c$, illustrating that enhancement of the gel point spans a range of mixing ratios.

In order to gain information regarding the microscopic mechanisms leading to this enhanced gel formation, we conducted x-ray photon correlation spectroscopy (XPCS) experiments on the suspensions. (See Appendix A for details regarding the experimental procedures.) Figure 3 shows the small-angle x-ray scattering (SAXS) intensity $I(Q)$ for the suspension with $c=0.5$ and $\phi_{T}=0.45$ at temperatures above and below $T^{*}$. The intensity includes two peaks in the measured $Q$ range. Similar features were observed in $I(Q)$ for mixtures with $c=0.5$ and $\phi_{T}=0.30$, as shown in Fig. 4(a). For comparison, Figs. 5(a) and 5(b) display $I(Q)$ for monodisperse suspensions of large and small colloids ( $c=0$ and $c=1)$ above and below $T^{*}$ at $\phi_{T}=0.45$ and 0.30 , respectively. Based on $I(Q)$ for the monodisperse suspensions and the measured form factors of the two colloids, we identify the two peaks in $I(Q)$ of the mixtures with peaks in the interparticle structure factor $S(Q)$ corresponding to near-neighbor correlations. Specifically, the first peak at $Q=0.18 \mathrm{~nm}^{-1}$ results from near-neighbor correlations of the larger colloids, and the second peak at $Q=0.32 \mathrm{~nm}^{-1}$ reflects near-neighbor correlations between the smaller colloids. In principle, one also expects a third peak in $S(Q)$ of the mixtures near $Q=0.25 \mathrm{~nm}^{-1}$ corresponding to the cross term from near-neighbor correlations between a large and a small colloid. Given the miscibility of the two colloidal species when they behave as hard spheres, we believe such a peak should be present in $S(Q)$ of the mixtures, at least at $T>T^{*}$. However, calculations of the structure factor of hard-sphere mixtures indicate that the third peak corresponding to large-small particle correlations is masked in $I(Q)$ by a

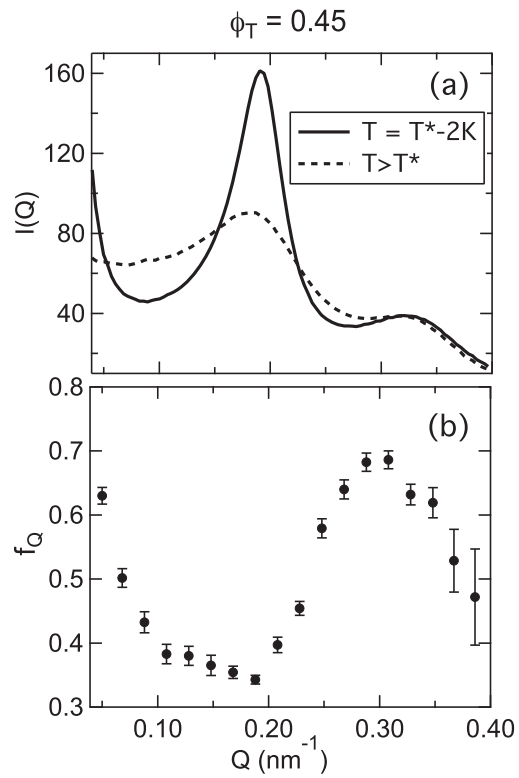

FIG. 3. (a) Scattering intensity of a binary mixture ( $\left.c=0.5, \kappa=2, \phi_{T}=0.45\right)$ of silica nanocolloids in the high-temperature fluid phase $(T=303 \mathrm{~K}$, dashed line) and low-temperature gel phase $(T=288 \mathrm{~K}$, solid line). (b) Nonergodicity parameter $f_{Q}$ in the gel phase determined with XPCS at $T=288 \mathrm{~K}$ $(\Delta T \approx-2 \mathrm{~K})$.

minimum in the form factor, ${ }^{47}$ which would explain its absence in Figs. 3(a) and 4(a). ${ }^{48}$

Nevertheless, comparisons of changes in $I(Q)$ upon gel formation in the mixtures versus monodisperse suspensions reveal differences in the microscopic mechanisms underlying gelation in each case. In particular, as seen in Fig. 5(a), at $\phi_{T}=0.45$, the monodisperse suspensions show remarkably little change in $I(Q)$ over the measured $Q$ range as a result of gel formation, consistent with earlier studies ${ }^{43,49,50}$ In contrast,

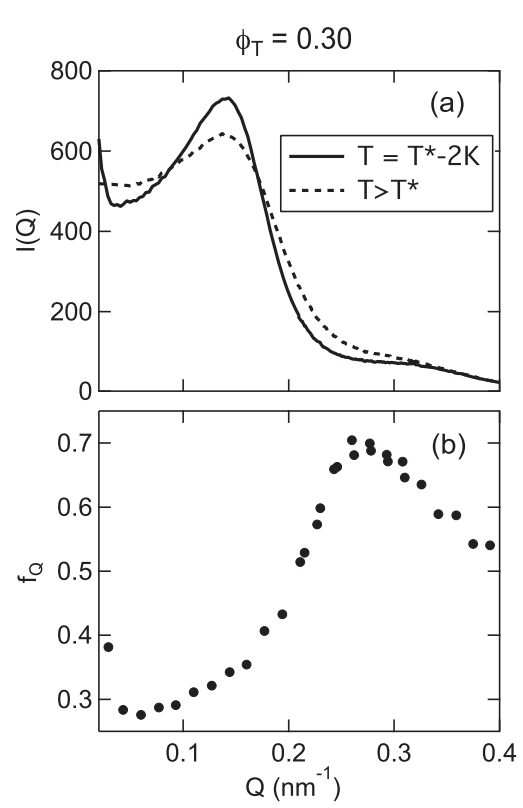

FIG. 4. (a) Scattering intensity of a binary mixture $\left(c=0.5, \kappa=2, \phi_{T}=0.3\right)$ of silica nanocolloids in the high-temperature fluid phase $(T=298 \mathrm{~K}$, dashed line) and low-temperature gel phase ( $T=283 \mathrm{~K}$, solid line). (b) Nonergodicity parameter $f_{Q}$ in the gel phase determined with XPCS at $T=283 \mathrm{~K}$ $(\Delta T \approx-2 \mathrm{~K})$. 


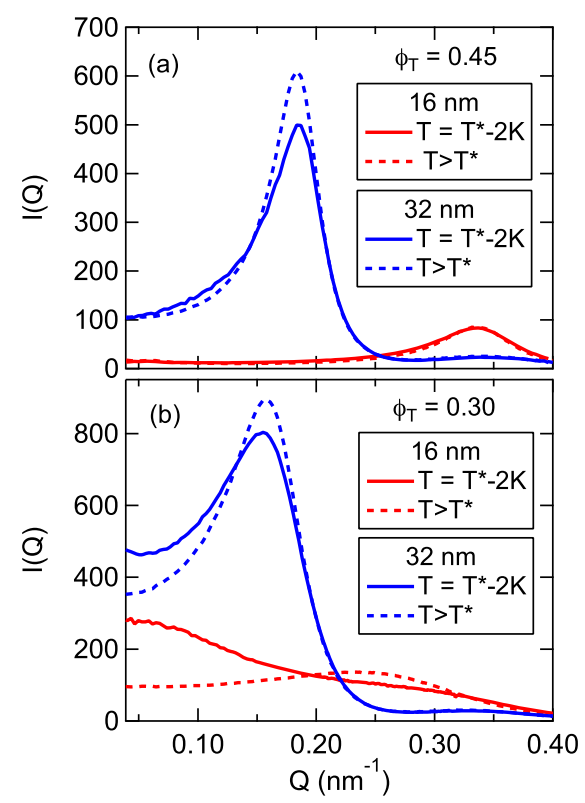

FIG. 5. (a) Scattering intensities of monodisperse suspensions with (a) $\phi_{T}=0.45$ and (b) $\phi_{T}=0.30$ in the high-temperature fluid phase (dashed lines) and low-temperature gel phase (solid lines). The blue curves correspond to suspensions of larger colloids $(c=0)$, and the red curves correspond to suspensions of smaller colloids $(c=1)$.

the peaks in $I(Q)$ of the mixture at $\phi_{T}=0.45$ become sharper and the low- $Q$ intensity increases on cooling into the gel phase, indicating an increase in large length-scale heterogeneity and an enhancement of near-neighbor correlations among samesized colloids. Similarly, the peaks become sharper in the mixture with $\phi_{T}=0.30$ upon gelation, as seen in Fig. 4(a), although at this lower concentration, both the mixtures and the monodisperse suspensions display increases in the low$Q$ intensity on cooling into the gel phases. (Indeed, we note that the low- $Q$ scattering becomes particularly pronounced in the small-colloid suspension with $R_{S} \approx 8 \mathrm{~nm}$, as seen in Fig. 5(b), a feature we speculate results from distinct features of the gel structure due to the larger range of attraction relative to particle radius in this case.) These changes are evidence that the particle attractions trigger micro-phase separation in the mixtures and further that this de-mixing drives the gelation, pre-empting the percolation driven "equilibrium gelation" that has been documented in the monodisperse suspensions. ${ }^{42,49}$ Recent theoretical work on colloid mixtures with a binary size distribution and repulsive interactions, ${ }^{51}$ as well as work on mixtures of same-sized colloids that are unstable to demixing due to asymmetric attractive interactions, ${ }^{9}$ has revealed similar micro-phase separation.

Figure 6 displays the XPCS intensity autocorrelation function $g_{2}(Q, t)$ at three wave vectors measured on a mixture with $c=0.5$ and $\phi_{T}=0.45 \mathrm{in}$ the gel state at $T=288 \mathrm{~K}$ (an undercooling of $\Delta T=T-T^{*} \approx-2 \mathrm{~K}$ ). Consistent with earlier XPCS studies on monodisperse suspensions, ${ }^{43,44}$ in the gel phase, $g_{2}(Q, t)$ has a terminal relaxation that is described by a compressed-exponential line shape,

$$
g_{2}(Q, t)=1+b\left(f_{Q} \exp \left[-(\Gamma t)^{\beta}\right]\right)^{2},
$$

where $b$ is the Siegert factor, which we determined from a separate measurement on a static silica aerogel sample to vary

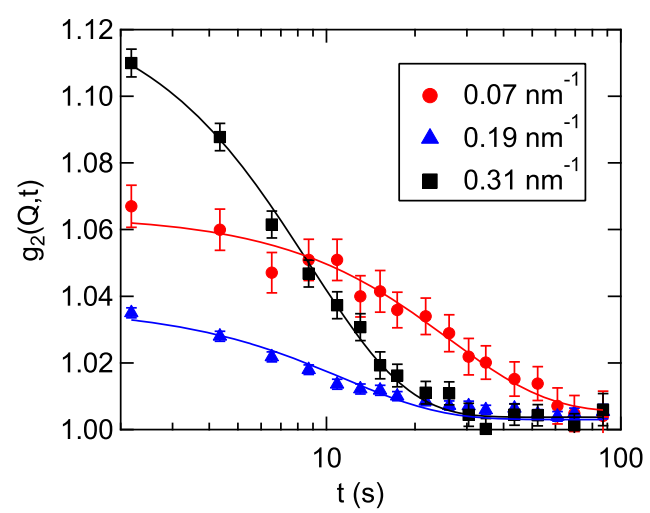

FIG. 6. XPCS intensity autocorrelation function of a binary mixture ( $c=0.5$, $\left.\phi_{T}=0.45, \kappa=2\right)$ of silica nanocolloids at $T=288 \mathrm{~K}(\Delta T \approx-2 \mathrm{~K})$ for three wave vectors, $Q=0.07 \mathrm{~nm}^{-1}$ (red circles), $0.19 \mathrm{~nm}^{-1}$ (blue triangles), and $0.31 \mathrm{~nm}^{-1}$ (black squares). The solid lines show the results of fits using Eq. (1) with $\beta=1.5$.

from $b \approx 0.31$ at $Q=0.04 \mathrm{~nm}^{-1}$ to $b \approx 0.29$ at $Q=0.4 \mathrm{~nm}^{-1}$. The solid lines in Fig. 6 are the results of fits to the data using Eq. (1), which gives $\beta \approx 1.5$ and a decay rate $\Gamma$ that varies with wave vector as $\Gamma \sim Q$. Such compressed-exponential correlation functions $(\beta>1)$ with $\Gamma \sim Q$ are signatures of slow, hyperdiffusive motion that has been widely observed with XPCS and dynamic light scattering on colloidal gels and other disordered soft solids and that has been identified with long length-scale strain motion in response to heterogeneous stress relaxation. ${ }^{52-55}$ In particular, these dynamics are not thought to be related to local, diffusive structural relaxation of the particles. Due to the sensitivity of the gels to radiation damage, we did not study extensively the behavior of this slow relaxation, which has been investigated in detail elsewhere, ${ }^{43}$ but instead focused on characterizing accurately the plateau value of $g_{2}(Q, t)$ prior to the terminal decay, quantified in Eq. (1) by the non-ergodicity parameter $f_{Q}$, a feature we could measure reliably with $\mathrm{x}$-ray exposures below those that cause measurable damage.

Specifically, $f_{Q}$ is a measure of the plateau amplitude of the electric field autocorrelation function, $g_{1}(Q, t)$, which is related to $g_{2}$ by the Siegert relation, $g_{2}=1+b g_{1}^{2}$. The electric field autocorrelation function is in turn equivalent to the coherent intermediate scattering function $F_{c}(Q, t)$, with each particle pair weighted by the product of their $\mathrm{x}$-ray form factors. The fact that the measured amplitudes of the decay of $g_{2}(Q, t)$ are less than $b$ implies a partial decay in the correlation function, and hence a degree of colloidal motion, on time scales that are too short to access in the measurement, as illustrated schematically in Fig. 7. Such multi-step decays are generic for many colloidal gels. In monodisperse concentrated gels, the magnitude of $f_{Q}$ displays peaks in $Q$ that track those in the static structure factor. ${ }^{24,43,56-58}$ This correspondence reflects the relationship between the constraints to collective localized motion in the dynamically arrested, nonergodic state and the spatial correlations of the colloids in the gel, and one can further extract a characteristic localization length from the $Q$-dependence of $f_{Q} \cdot{ }^{43,58}$

Figure 3(b) shows an example of $f_{Q}$ for $c=0.5$ and $\phi_{T}=0.45$ in the gel state at $T=288 \mathrm{~K}(\Delta T \approx-2 \mathrm{~K})$. Interestingly, $f_{Q}$ tracks some but not all of the features in $S(Q)$. 


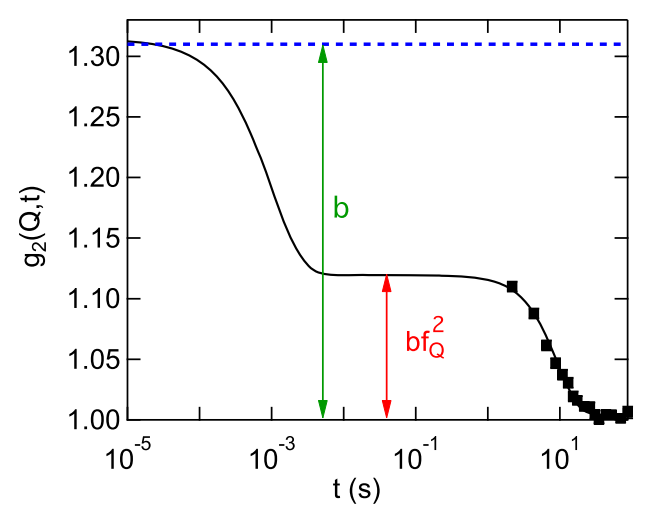

FIG. 7. Putative picture of a multi-step decay in the intensity autocorrelation function of a colloidal gel. The black squares are the data for the mixture ( $\left.c=0.5, \phi_{T}=0.45, \kappa=2\right)$ at $Q=0.31 \mathrm{~nm}^{-1}$ from Fig. 6 . The solid line is a simple exponential decay representing schematically the dynamics at short delay times, smoothly connected with a plateau region followed by the compressed-exponential form that describes the measured terminal relaxation.

Specifically, the non-ergodicity parameter displays a peak coincident with the second peak in $I(Q)$ corresponding to correlations between the smaller colloids but is negligible in the region of the first peak in $I(Q)$ corresponding to correlations between the larger colloids. This finding is true also for $\phi_{T}=0.30$, as seen in Fig. 4(b), which displays $f_{Q}$ for $c=0.5$ and $\phi_{T}=0.30$ at $\Delta T \approx-2 \mathrm{~K}$. In contrast to the nonergodicity parameter for monodisperse gels, the results in Figs. 3(b) and 4(b) indicate a striking dynamic heterogeneity in the mixtures. Specifically, we hypothesize that they imply that gel formation in the mixtures results from the structural arrest of the smaller particles while the larger particles remain mobile, exactly the opposite scenario from depletion-driven gelation of binary mixtures!

To test this hypothesis and to elucidate further the microscopic processes driving dynamic arrest in binary colloidal mixtures with short-range attraction, we conducted molecular dynamics simulations of colloids with total volume fraction $\phi_{T}=0.30$ evenly divided by volume fraction between spheres with average radii $R_{L}=16 \sigma$ and $R_{S}=8 \sigma$, where $\sigma$ is a characteristic simulation length scale. Several sets of simulations were performed with varying polydispersities about these averages. The particles experienced pairwise interactions that combined two contributions (see Appendix A for details): (i) a hard repulsive potential that diverged on contact and that decayed steeply with increasing separation in a Yukawa form with energy scale $\epsilon$ and with decay length $\xi=\sigma / 10$ and (ii) a tunable short-range attraction modeled as a thin Gaussian well of width $\sigma$ and depth that increased linearly with the temperature below $T_{\theta}=\epsilon / k_{B}$. This combination of terms mimics the potential of the octadecyl-silica nanocolloids in the experiment, while providing a smooth analytic form that facilitates the simulations. We found that modifying the precise shape of the potential did not qualitatively change the simulation results.

Figures $8(a)-8(c)$ display the self-intermediate scattering function $F_{s}(Q, t)$ for the large and small particles at $Q=0.575 / \sigma$ calculated from their trajectories at a set of temperatures in a simulation with $3 \%$ polydispersity on both the large and small species. At the highest temperature shown,

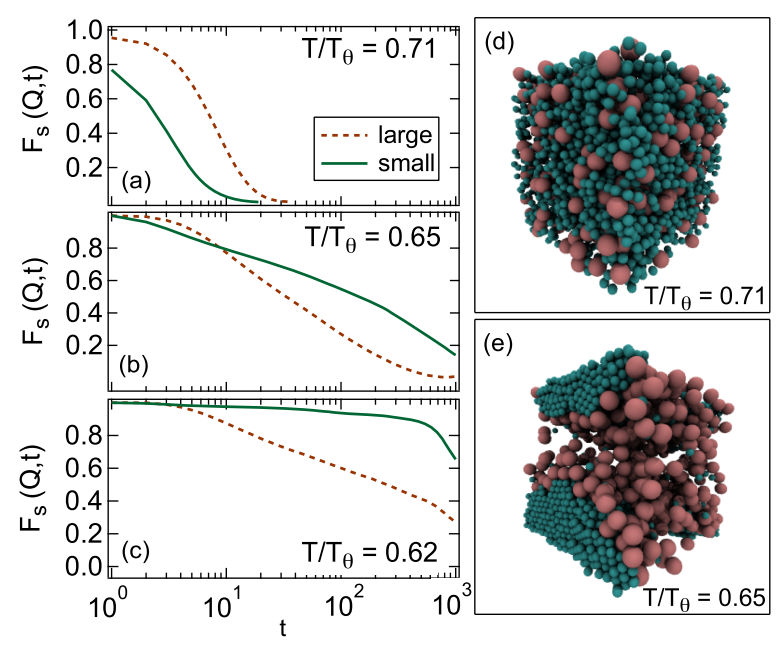

FIG. 8. Molecular dynamics simulation results of a binary mixture of colloids $\left(\phi_{T}=0.3, c=0.5, \kappa=2\right)$ with a short-range attraction strength that increases linearly with decreasing temperature. (a)-(c) display the self-intermediate scattering function $F_{s}(Q, t)$ for the large (red dashed lines) and small (green solid lines) particles at $Q=0.575 / \sigma$ at three temperatures traversing the ergodic-to-nonergodic transition in the mixture; (a) $0.71 T_{\theta}$, (b) $0.65 T_{\theta}$, and (c) $0.62 T_{\theta}$. (d) shows an image of the instantaneous particle positions at a high temperature in the ergodic fluid phase. (e) shows an example of the particle positions at a temperature below the transition.

$T=0.71 T_{\theta}$ [Fig. 8(a)], $F_{s}(Q, t)$ decays quickly in a manner consistent with liquid-state dynamics. As expected for liquids, $F_{s}(Q, t)$ of the smaller particles decays more rapidly than that of the larger particles due to the smaller particles' greater mobility. Consistent with these dynamics, the microstructure resembles that of homogeneously mixed fluid, as illustrated in Fig. 8(d), which displays an image showing the instantaneous particle positions at this temperature.

At slightly lower temperatures, however, the microstructure and dynamics change dramatically. The change in dynamics is illustrated in Figs. 8(b) and 8(c), which show $F_{s}(Q, t)$ at $T=0.65 T_{\theta}$ and $T=0.62 T_{\theta}$, respectively. Due to the increasing interparticle attractions, the particle dynamics at these temperatures are slowed considerably. Moreover, the slowing is much more pronounced among the smaller particles. Eventually, these dynamics become too slow to conduct proper averaging on the time scale of the simulations. Thus, the simulations mirror the experiments in that non-ergodicity is driven by dynamic arrest of the smaller particles. The microstructural origin of this arrest is illustrated in Fig. 8(e), which shows an image of the instantaneous particle positions at $T=0.65 T_{\theta}$. The system has undergone phase separation in which the arrested smaller particles have formed monodisperse, high-density regions that are interspersed among lowerdensity regions populated predominantly by the mobile larger particles.

\section{DISCUSSION}

These simulation and XPCS results together reveal the microscopic processes leading to gel formation in the binary mixtures of colloids with short-range attraction. Specifically, while the particles of two sizes are miscible when their interactions are hard-sphere-like or only weakly attractive, for 
stronger attraction, they become immiscible. Hence, as a result of increasing attraction, the smaller particles segregate into dense regions where they undergo dynamical arrest that halts the phase separation. In the simulations, this arrest is accompanied by the formation of local crystalline order, as seen in Fig. 8(e). In the experiments, no evidence of crystal formation is apparent in $I(Q)$. We believe that this difference between the simulations and experiments in the local order of the small colloids at low temperature is a consequence of the different polydispersity in the small-colloid population in the two cases. Due to the higher polydispersity in the experiments, the locally dense regions of smaller colloids remain amorphous, and we associate their dynamical arrest with formation of a dense gel or attractive glass phase that imparts mechanical rigidity to the system. $^{24}$

To understand the mechanisms driving this gel-formation scenario, comparison with gel formation in monodisperse suspensions of colloids with short-range attraction is instructive. In the case of monodisperse suspensions, the introduction of attractive interactions makes the system unstable to liquidgas phase separation (see Appendix B). The ensuing spinodal decomposition is a path to gelation, as the colloids can condense into percolating regions that vitrify, thereby arresting the phase separation. ${ }^{1}$ In the case of a mixture, a second instability with respect to compositional demixing is also possible, and indeed the partitioning of the smaller colloids into dense regions indicates that the system is unstable to composition fluctuations, while the samples' optical translucency suggests that any instability does not result in large-scale phase separation.

Demixing as a route to bigel formation has been shown experimentally in binary mixtures of same-sized colloids $(\kappa=1)$ in which the inter-species attraction is weaker than the intra-species attraction. ${ }^{9}$ These results were further supported by thermodynamic perturbation theory (TPT) calculations of the mixture instability to compositional demixing. ${ }^{9}$ Interestingly, we have performed comprehensive TPT calculations (details given in Appendix B) that do not identify compositional demixing as the dominant instability for bidisperse mixtures with a moderate size ratio $(\kappa=2)$ and otherwise symmetric interactions. Rather, the spinodal boundary of the binary mixtures in this case corresponds solely to a condensation-type instability at a thermodynamic level. These calculations hence indicate that the mechanisms promoting the demixing seen in the experiments and simulations are not captured by such a mean-field model, likely as a consequence of fluctuations due to subtle entropic packing effects. Specifically, because the range of attraction is the same for the large and small colloids on an absolute scale, the range divided by the particle radius is larger for the smaller particles. We speculate that this difference could lead to more configurations among the smaller particles in which many small particles are in each others' attractive wells that ultimately give an advantage to the de-mixing and dynamic arrest.

\section{CONCLUSION}

The unexpected behaviour of weakly attractive binary mixtures presented here illustrates how tuning the miscibility of mixtures through their interparticle interactions can lead to new routes for controlling the rheology and phase behavior of suspensions. The findings suggest several directions to explore this theme further. For instance, while the study here focused on the colloid size ratio $\kappa=2$, an interesting question is how the gel formation behavior evolves as this ratio is varied. Specifically, how do the miscibility of the two sizes and the role of each species in dynamic arrest change on increasing the ratio toward $\kappa \approx 5$, where depletion effects dominate? Also, how does the gel formation process change as the ratio is tuned toward the monodisperse case, $\kappa=1$, where previous work has shown that gelation in these nanocolloidal suspensions with short-range attraction is driven by percolation ${ }^{42,49}$ ? Finally, while the details of the process we have uncovered are particular to the mixtures studied here, this enhancement of the fluid-gel transition temperature resembles findings in other multicomponent fluid systems. For example, binary mixtures of molecular glass formers can display a glass transition temperature $T_{g}$ that is higher than that expected from an ideal mixing law ${ }^{59,60}$ and in some cases is even higher than $T_{g}$ of either pure component. ${ }^{61}$ Also, enhanced aggregation upon binary mixing of eye-lens proteins with short-range attraction has been implicated in some forms of cataract. ${ }^{62}$ Thus, the phenomena illustrated by these nanocolloidal mixtures potentially serve as a model for understanding fluid-solid transitions in a range of multicomponent disordered materials.

\section{ACKNOWLEDGMENTS}

We gratefully acknowledge D. Berman, S. Narayanan, and A. Sandy for their expert assistance. The research was supported by NSERC Discovery \& CREATE grants, the NSF under Grant Nos. CBET-1336166 and HRD-1238524, funding from EMBO (ALTF181-2013), and an ERC Advanced Grant MiCE (No. 291234). The use of the APS was supported by DOE BES under Contract No. DE-AC02-06CH11357.

\section{APPENDIX A: MATERIALS AND METHODS}

\section{Sample preparation}

Silica nanocolloids with average radii of 8 and $16 \mathrm{~nm}$ (with $10 \%$ polydispersity), as determined by SAXS on dilute suspensions, were prepared using the Stober method of basecatalyzed hydrolysis and condensation of tetraethyl orthosilicate. ${ }^{63,64}$ The octadecyl surface functionalization followed the method of van Helden, Jansen, and Vrij. ${ }^{65}$ Suspensions of each species of colloid were prepared by dispersing a known mass of dried silica powder in decalin by gentle stirring, and these suspensions were then combined at temperatures well above $T^{*}$ to form initially homogeneous binary mixtures with the desired particle concentrations.

\section{XPCS}

Experiments were conducted at Sector 8-ID-I of the Advanced Photon Source using $7.35 \mathrm{keV}$ x-rays. Details regarding the beamline and measurement procedures have been presented elsewhere. ${ }^{66,67}$ Samples were contained in sealed stainless-steel holders with sample thickness $0.5 \mathrm{~mm}$ 
and with polyimide windows for transmission scattering. The coherent scattering intensity, recorded by a direct-illuminated CCD area detector, was measured over the range of wavevector magnitudes $0.04 \mathrm{~nm}^{-1}<Q<0.40 \mathrm{~nm}^{-1}$. Timeresolved series of scattering intensities $I\left(Q, t^{\prime}\right)$ were analyzed to determine the intensity time-autocorrelation function,

$$
g_{2}(Q, t)=\frac{\left\langle I\left(\boldsymbol{Q}, t^{\prime}\right) I\left(\boldsymbol{Q}, t^{\prime}+t\right)\right\rangle}{\left\langle I\left(\boldsymbol{Q}, t^{\prime}\right)\right\rangle\left\langle I\left(\boldsymbol{Q}, t^{\prime}+t\right)\right\rangle},
$$

where the brackets indicate a time average over time $t^{\prime}$ and an ensemble average over equivalent detector pixels at the same wave-vector magnitude $Q$ to assure proper ensemble averaging for the non-ergodic gels. Exposure times of $0.25 \mathrm{~s}$ were employed, and the beam was shuttered between exposures to reduce sample irradiation. The total exposure in any measurement was limited to $12.5 \mathrm{~s}$ (i.e., $50 \mathrm{CCD}$ images) to avoid measurable effects of radiation damage on $g_{2}(Q, t)$. The resulting range of delay times, limited at short times by the CCD frame rate, was approximately $2.1 \mathrm{~s}<t<100 \mathrm{~s}$. As mentioned above, this range was not always adequate to cover the slow terminal relaxation of $g_{2}(Q, t)$ in the gel state but was sufficient to determine accurately the plateau value and hence the non-ergodicity parameter $f_{Q}$.

\section{Rheometry}

Measurements of the frequency-dependent complex shear modulus were performed using a stress-controlled rheometer (Anton-Paar MCR 300) in a cone-plate geometry with a frequency of $\omega=1 \mathrm{~s}^{-1}$ and a strain amplitude, $\gamma=0.3 \%$, that was well within the linear viscoelastic regime. The rheometer was equipped with a Peltier device for temperature control, which was calibrated against the thermometry employed in the XPCS experiments. The cup-shaped bottom plate was fashioned with a reservoir containing a $50 \%$ ethylene glycol/water solution around the tool to prevent the evaporation of the decalin, making the samples stable in the rheometer for several days.

\section{Molecular dynamics simulations}

Simulations were conducted using the HOOMD-Blue package. ${ }^{68,69}$ The simulation system was composed of approximately 4000 small particles $\left(R_{S}=8 \sigma\right)$ and 500 large particles $\left(R_{L}=16 \sigma\right)$, where $\sigma$ is a characteristic simulation length scale, maintained at volume fraction $\phi=0.3$ in a cubic simulation box with periodic boundary conditions. Simulations were performed for several values of particle size polydispersities. As the particles were of the same material, their characteristic masses were $M_{S}=m$ and $M_{L}=8 m$. The interparticle potential was the sum of a repulsive Yukawa potential $U_{\text {rep }}$ and an attractive Gaussian well $U_{\text {att }}$ of the form

$$
\begin{aligned}
& U_{\text {rep }}\left(r_{i j}\right)= \begin{cases}\frac{\epsilon \exp \left[-\left(r_{i j}-\Delta_{i j}\right) / \xi\right]}{r_{i j}-\Delta_{i j}} & \text { if } r_{i j}<r_{c u t}+\Delta_{i j}, \\
0 & \text { otherwise }\end{cases} \\
& U_{a t t}\left(r_{i j}\right)= \begin{cases}U_{0}(T) \exp \left[-2 \frac{\left(r_{i j}-\Delta_{i j}\right)^{2}}{\sigma^{2}}\right] & \text { if } r_{i j}<r_{c u t}+\Delta_{i j} \\
0 & \text { otherwise }\end{cases}
\end{aligned}
$$

where $\epsilon=k_{B} T_{\theta}$ is the characteristic energy scale, $U_{0}(T)$ $=10\left(k_{B} T-\epsilon\right)$ is the temperature-dependent strength of the attractive Gaussian potential, $\xi=\sigma / 10$ is the range of the Yukawa potential, $\Delta_{i j}=R_{i}+R_{j}$ is the contact separation of a given pair of particles (with $i, j \in\{S, L\}$ ), and $r_{c u t}=3.5 \sigma$ is the cutoff for both the Yukawa and the Gaussian potentials. Thus, the corresponding characteristic simulation time scale was $\tau=\sqrt{m \sigma^{2} / \epsilon}$. Particle trajectories were calculated by integrating the equations of motion using a time step of $\Delta t=0.005 \tau$. The simulations proceeded by cooling slowly from a high initial temperature, $T=0.8 T_{\theta}$, where the particles remained well mixed and freely diffusing, stopping in increments of $\Delta T=0.03 T_{\theta}$ to explore the dynamics. During the temperature ramps between set points, which lasted $1 \times 10^{5}$ steps, the system evolved in the canonical (NVT) ensemble using a Nosé-Hoover thermostat to couple the system to a heat bath. After reaching each target temperature, further $1 \times 10^{5}$ integration steps in the NVT ensemble were performed, after which the system evolved in the microcanonical (NVE) ensemble for $2 \times 10^{7}$ steps during which data were acquired. Eventually, at some temperature, the structural dynamics became extremely slow so that equilibration on these time scales was impossible, and the system became effectively nonergodic. The self-part of the Van Hove correlation function $G_{s}(r, t)$ was calculated for the small and large particle populations from the distribution of mean-square particle displacements at various time intervals $t$. The normalized, selfpart of intermediate scattering function $F_{s}(Q, t)$ for the small and large particles was then obtained via the spatial Fourier transform of these correlation functions.

\section{APPENDIX B: THERMODYNAMIC PERTURBATION THEORY}

Here we employ perturbation theory ${ }^{70}$ to calculate thermodynamic predictions for the stability of the binary octadecyl-coated nanocolloidal mixture. In particular, we are interested in determining whether thermodynamic perturbation theory (TPT) predicts an instability that is dominated by density or composition fluctuations, i.e., a principally condensation- or demixing-type instability. The nature of the instability can be ascertained from the eigenvalues of the stability matrix

$$
\boldsymbol{F}=\left[\begin{array}{ll}
f_{v v} & f_{v x} \\
f_{x v} & f_{x x}
\end{array}\right]
$$

where $f_{v x}=\left.\frac{1}{2} \frac{\partial^{2} f}{\partial v \partial x}\right|_{T}, f_{v v}=\left.\frac{1}{2} \frac{\partial^{2} f}{\partial v^{2}}\right|_{T, x}$, etc., are the second derivatives of the reduced Helmholtz free energy $f$ with respect to the overall specific volume $v=1 / \rho=V /\left(N_{S}+N_{L}\right)$ and composition as measured by the molar fraction of small colloids $x=N_{S} /\left(N_{S}+N_{L}\right)$ at fixed temperature $T$.

Thermodynamic stability is bound by the spinodal surface, as given by $\operatorname{det}[\boldsymbol{F}]=0$, and the relative significance of composition and density fluctuations is measured by the angle

$$
\alpha=\arctan \left(\frac{f_{v x}}{f_{v v}-\lambda_{-}}\right),
$$


where $\lambda_{-}$is the smallest eigenvalue of the stability matrix. ${ }^{9,71}$ A predominantly demixing-type instability is indicated by $\alpha \rightarrow 0$, while $\alpha \rightarrow \pm \pi / 2$ suggests a condensation-type instability.

The free energy is found by assuming that the silica nanocolloids are well approximated as hard, spherically symmetric spheres and modeling the attractive octadecyl surface layers as short-ranged, pairwise potentials. The mixture's free energy $f=f^{0}+f^{\mathrm{P}}$ is then calculated as a perturbation $f^{\mathrm{P}}$ about the known binary hard-sphere mixture $f^{0}$.

\section{Colloidal hard-sphere reference}

The reference free energy $f^{0}$ is the sum of the ideal free energy $f^{\text {id }}$, which can be computed directly from the ideal partition function for a multi-component gas, and the hardsphere free energy $f^{\mathrm{HS}}$. A mixture of hard spheres obeys the Boublík-Mansoori-Carnahan-Starling-Leland equation of state and has a well-known analytical form for the hard-sphere free energy. ${ }^{72-74}$

\section{Attractive perturbation}

The hard-sphere repulsive potential is perturbed by an attractive short-ranged potential $U_{i j}^{\mathrm{P}}(\boldsymbol{r})$ between pairs of colloids $i, j \in\{S, L\}$. In the molecular dynamics simulations, the attraction is modeled as a spherically symmetric Gaussian well. In the TPT calculations, we use the even simpler pairwise square-well,

$$
U_{i j}^{\mathrm{P}}(r)= \begin{cases}-u_{i j} & \text { if } \sigma_{i j}<r<\sigma_{i j}+\lambda_{i j} \\ 0 & \text { otherwise }\end{cases}
$$

with $i, j \in\{S, L\}$ for interaction ranges $\lambda_{i j}$, effective diameters $\sigma_{i j}=R_{i}+R_{j}$, and well depths $u_{i j}$.

In the molecular dynamics simulations presented above, the well depth varied linearly with the temperature in an identical manner for both large and small colloids. Here, we use a generalized linear form: $u_{i j}(T)=\omega_{i j}\left(u \epsilon-v k_{\mathrm{B}} T\right)$, in which $\epsilon$ defines the characteristic energy scale of the attractive well, $\omega_{i j}$ controls the strength of pairwise interactions between different species, and the dimensionless numbers $u$ and $v$ set the relative importance of the attractive and thermal energies. In the TPT calculations, thermal energy is given in units of $\epsilon$. Likewise, we choose the radius of the larger colloids $R_{L}=2 R_{S}=16 \mathrm{~nm}$ as the characteristic length scale, and introduce the nondimensional length scales $\tilde{R}_{i}=R_{i} / R_{L}, \tilde{\sigma}_{i j}=\left(R_{i}+R_{j}\right) / R_{L}$, and $\tilde{\lambda}_{i j}=\lambda_{i j} / R_{L}$.

The first-order term in the expansion of the free energy is the sum over all pairs of convolutions of the perturbation potentials $U_{i j}^{\mathrm{P}}$ and unperturbed partial radial distribution functions $g_{i j}^{0}$ of the reference hard-sphere mixture,

$$
f^{\mathrm{P}}=\frac{\rho}{2 k_{\mathrm{B}} T} \sum_{i, j=1}^{2} x_{i} x_{j} \int g_{i j}^{0}(\boldsymbol{r}) U_{i j}^{\mathrm{P}}(\boldsymbol{r}) d \boldsymbol{r},
$$

where $x_{i} \equiv N_{i} /\left(N_{S}+N_{L}\right)$ for $i \in\{S, L\}$. The unperturbed partial radial distribution functions $g_{i j}^{0}$ for binary hard-spheres are found through the Percus-Yevick approximation for the Ornstein-Zernike equation, which produces analytical forms in reciprocal space of the total correlation functions. These depend on the direct correlation functions, ${ }^{75}$ which are known for binary mixtures of hard spheres. ${ }^{47,76}$ Strong oscillations in the inverse Fourier transform of the total correlation functions can arise due to the hard-sphere discontinuities. To remedy such oscillations, the discontinuity is removed by subtracting the contact value everywhere beyond the contact point in real space, after adding its Fourier transform to the reciprocal space function prior to the inverse transformation. ${ }^{75}$ Additionally, the Grundke-Henderson correction is applied to the PercusYevick approximations for $g_{i j}^{0}$, since their values and slopes are otherwise under-predicted at the contact point. ${ }^{77-79}$

\section{TPT results}

In the bidisperse mixture of octadecyl-coated silica colloids, we experimentally observe that the colloidal gels form at higher temperatures than either pure suspensions and that the smaller colloids demix, undergoing dynamic arrest while the larger particles remain mobile. To give context for this behaviour, we present TPT predictions for four cases of binary mixtures. The first three cases (1-3) are binary mixtures of monodisperse suspensions, in which intra- and inter-species interactions are asymmetric. TPT calculations of these three cases show that a region exists on the spinodal surface for which composition fluctuations are dominant, indicating that the suspensions are unstable to demixing. In fact, case 1 essentially reproduced the results of Varrato et al. ${ }^{9}$ However, in the fourth case, we consider a bidisperse mixture with otherwise symmetric interactions that corresponds to our experimental system, for which we find that TPT does not predict the demixing instability that occurs experimentally. The details for these four cases are as follows:

1. A monodisperse suspension, in which the attractive potentials are independent of temperature and the interspecies well depth is reduced compared to the intraspecies depth. For all pairs, the effective diameter is $\tilde{\sigma}_{i j}=2$ (corresponding to $16 \mathrm{~nm}$ radius particles in contact) and the interaction range, $\tilde{\lambda}_{i j}=1 / 16$, is set by a species-independent thickness of the octadecyl chain coatings, $\lambda_{i j}=1 \mathrm{~nm}$. For this example, the intra-species attraction is $\omega_{i i}=1$, while the inter-species attraction is halved to $\omega_{i \neq j}=1 / 2$. The attraction is made independent of temperature by setting $u=1$ and $v=0$.

Figure 9(a) shows that a portion of the spinodal surface, $T^{*}\left(c, \phi_{T}\right)$, can exhibit demixing-type instabilities with $\alpha \approx 0$. Interestingly, as was experimentally observed for our octadecyl-coated nanocolloids and for the DNAcoated colloids of Varrato et al. ${ }^{9}$ we find that at sufficiently high volume fractions, an initially homogeneous symmetric mixture of $c \approx 0.5$ is most unstable toward demixing.

2. A monodisperse suspension, in which the interspecies well depth is reduced compared to the intra-species depth with linear temperature dependence. This is the same as case 1, except possessing a linear temperature dependence to account for the roughly linear increase in attraction with decreasing temperature. To match molecular dynamics simulations, we set $u=v=10$. Increasing $v$ from zero causes the spinodal curves to rise more quickly 

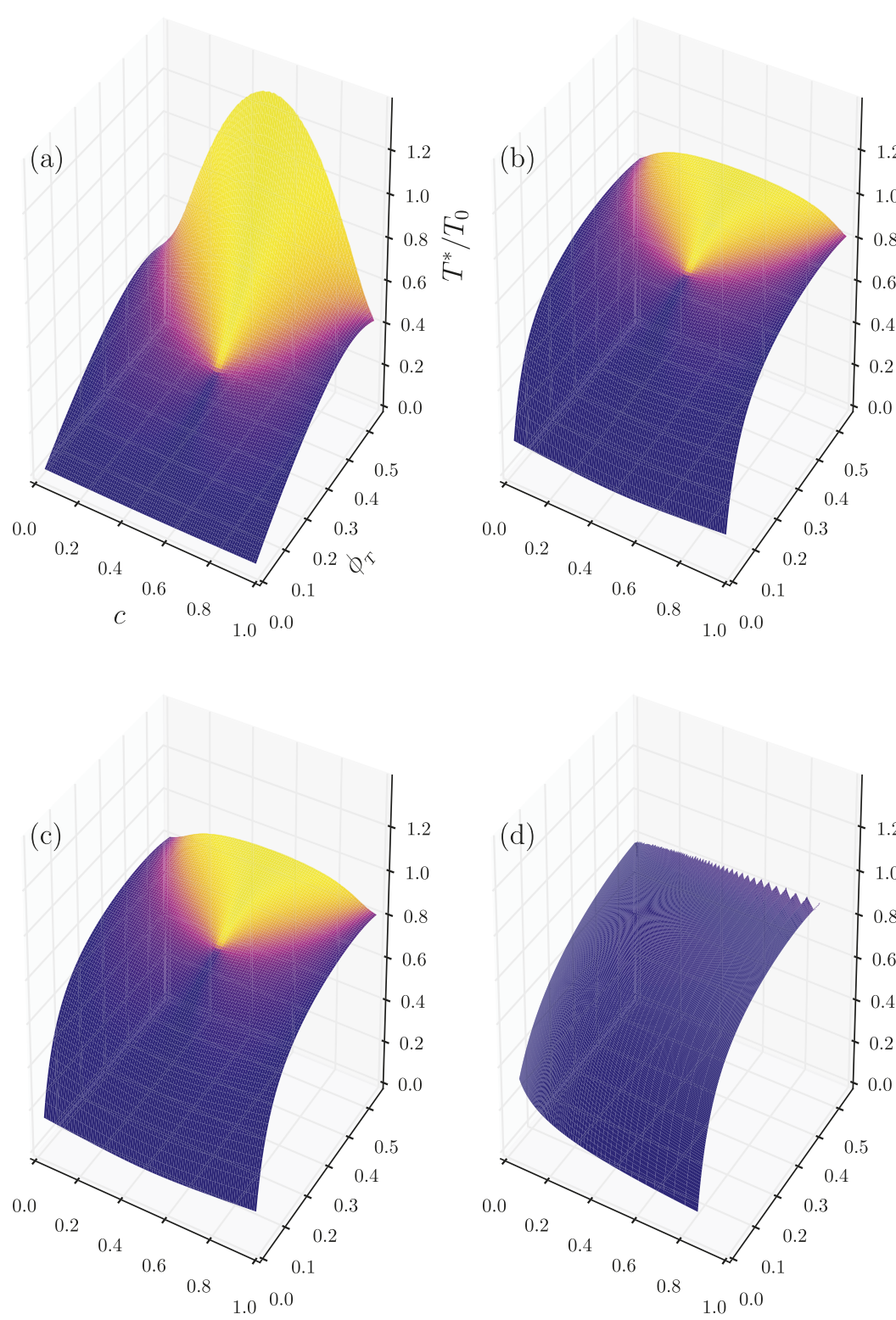

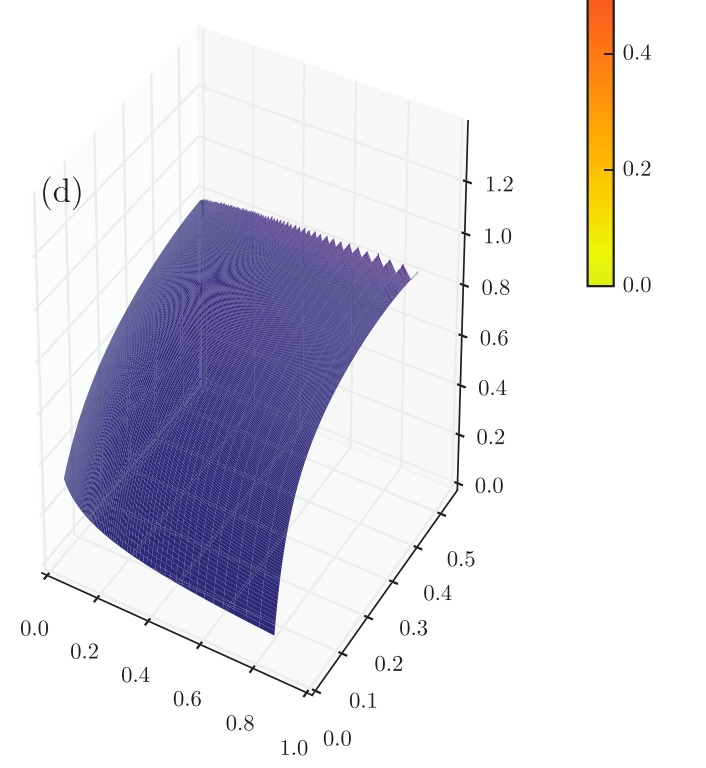

FIG. 9. Spinodal surfaces, $T^{*}\left(c, \phi_{T}\right)$, scaled by $T_{0}=\epsilon / k_{\mathrm{B}} T$, and stability parameter $\alpha$ predicted by TPT for four cases of binary mixtures. The first three [(a)-(c)] are monodisperse systems with binary interactions, while the fourth (d) is a bidisperse suspension with symmetric interactions. (a) A monodisperse suspension with binary well depths $\left(\omega_{i i}=1\right.$ and $\left.\omega_{i \neq j}=1 / 2\right)$. Interaction ranges are constant $\left(\tilde{\lambda}_{i j}=1 / 16\right)$ and independent of (b) A monodisperse suspension with linear temperature dependence $(u=v=10)$ and binary well depths $\left(\omega_{i i}=1\right.$ and $\left.\omega_{i \neq j}=1 / 2\right)$. Well ranges are constant $\left(\tilde{\lambda}_{i j}=1 / 16\right)$. (c) A monodisperse suspension with binary interaction ranges $\left(\tilde{\lambda}_{i i}=1 / 16\right.$ and $\tilde{\lambda}_{i \neq j}=1 / 32$ ). Well depth is species independent $\left(\omega_{i j}=1\right)$ and linearly dependent on temperature $(u=v=10)$. (d) Bidisperse suspension with radii $\tilde{R}_{L}=1$ and $\tilde{R}_{S}=1 / 2$ and otherwise symmetric interactions. Attraction is species independent $\left(\omega_{i j}=1\right.$ and $\tilde{\lambda}_{i j}$ $=1 / 16)$ and temperature dependent $(u=v=10)$. at small $\phi_{T}$ and then more slowly at large $\phi_{T}$, as shown in Fig. 9(b). Additionally, the temperature dependence diminishes the spinodal maximum of the symmetric mixture $(c \approx 0.5)$ with respect to the pure suspensions but does not erase it completely.

3. A monodisperse suspension, in which the interspecies interaction range is reduced compared to the intraspecies range with a linear temperature dependence. For all pairs, the effective diameter is $\tilde{\sigma}_{i j}=2$ and the well depth is $\omega_{i j}=1$, while the range is $\tilde{\lambda}_{i i}=1 / 16$ for intra-species attraction but only $\tilde{\lambda}_{i \neq j}=1 / 32$ for interspecies attraction. Temperature dependence is linear with $u=v=10$.

Just as in the previous case 2, demixing-type instabilities are possible in this case. Figure 9(c) shows that a portion of the spinodal surface can exhibit demixingtype instabilities with $\alpha \approx 0$ around a symmetric composition at sufficiently high volume fractions. As in case 2, the interactions favour intra-species over crossspecies attraction, which increases the significance of composition fluctuations relative to density fluctuations on the stability.

4. A bidisperse suspension with otherwise symmetric interactions and linear temperature dependence. These TPT calculations correspond most closely to the experimental system discussed in the main text. In contrast to the monodisperse examples, the mixture is binary but the interactions remain symmetric. Thus, both large $\left(\tilde{R}_{L}=1\right)$ and small $\left(\tilde{R}_{S}=1 / 2\right)$ colloids have equal well depths $\omega_{i j}=1$, equal interaction ranges $\tilde{\lambda}_{i j}=1 / 16$ for all pairs $(\forall i, j)$ and linear temperature dependence with $u=v=10$.

In this case, the instability boundary, shown in Fig. 9(d), is markedly different than cases 1 through 3 . There is no region of the spinodal surface that is primarily controlled by demixing-type instabilities. Instead, density fluctuations dominate everywhere on the surface. From a thermodynamic perspective, this is sensible since the interactions are symmetric and there is nothing to favour intra-species over inter-species interactions. Likewise, 
even in the absence of temperature dependence (results not shown), TPT does not predict a region dominated by demixing-type instabilities on the spinodal surface. These TPT predictions stand in stark contrast to the experimental and computational results presented in the main text, which are more indicative of microphase demixing than a condensation-type instability, suggesting a more complicated transition involving fluctuations in particle packing that cannot be incorporated in the TPT approach.

${ }^{1}$ E. Zaccarelli, "Colloidal gels: Equilibrium and non-equilibrium routes," J. Phys.: Condens. Matter 19, 323101 (2007).

${ }^{2}$ V. Trappe and P. Sandkuhler, "Colloidal gels-low-density disordered solidlike states," Curr. Opin. Colloid Interface Sci. 8, 494 (2004).

${ }^{3}$ J. A. Lewis, "Colloidal processing of ceramics," J. Am. Ceram. Soc. 83, 2341 (2000).

${ }^{4}$ M. L. Fisher and F. F. Lange, "Rheological behavior of slurries and consolidated bodies containing mixed silicon nitride networks," J. Am. Ceram. Soc. 83, 1861 (2000).

${ }^{5}$ G. V. Franks and F. F. Lange, "Plastic flow of saturated, consolidated alumina powder compacts: Particle size and binary mixtures," J. Am. Ceram. Soc. 82, 1595 (1999).

${ }^{6}$ A. Y. Mirarefi, S. Boutet, S. Ramakrishnan, A. J. Kiss, C.-H. C. Cheng, A. L. DeVries, I. K. Robinson, and C. F. Zukoski, "Small-angle x-ray scattering studies of the intact eye lens: Effect of crystallin composition and concentration on microstructure," Biochim. Biophys. Acta 1800, 556 (2010).

${ }^{7}$ E. R. Russell, J. Sprakel, T. E. Kodger, and D. A. Weitz, "Colloidal gelation of oppositely charged particles," Soft Matter 8, 8697 (2012).

${ }^{8}$ D. de las Heras, J. M. Tavares, and M. M. T. da Gama, "Bicontinuous and mixed gels in binary mixtures of patchy colloidal particles," Soft Matter $\mathbf{8}$, 1785 (2012).

${ }^{9}$ F. Varrato, L. Di Michele, M. Belushkin, N. Dorsaz, S. H. Nathan, E. Eiser, and G. Foffi, "Arrested demixing opens route to bigels," Proc. Natl. Acad. Sci. U. S. A. 109, 19155 (2012)

${ }^{10}$ R. Fantoni, D. Gazzillo, and A. Giacometti, "Thermodynamic instabilities of a binary mixture of sticky hard spheres," Phys. Rev. E 72, 011503 (2005).

${ }^{11}$ A. Jamnik, "Simulating asymmetric colloidal mixture with adhesive hard sphere model," J. Chem. Phys. 128, 234504 (2008).

${ }^{12}$ R. Pandey and J. C. Conrad, "Gelation in mixtures of polymers and bidisperse colloids,” Phys. Rev. E 93, 012610 (2016).

${ }^{13}$ W. C. K. Poon and P. B. Warren, "Phase-behavior of hard-sphere mixtures," Europhys. Lett. 28, 513 (1994).

${ }^{14}$ T. Biben and J. P. Hansen, "Phase-separation of asymmetric binary hardsphere fluids," Phys. Rev. Lett. 66, 2215 (1991).

${ }^{15}$ A. D. Dinsmore, A. G. Yodh, and D. J. Pine, "Phase-diagrams of nearly hard-sphere binary colloids," Phys. Rev. E 52, 4045 (1995).

${ }^{16}$ P. D. Kaplan, J. L. Rouke, A. G. Yodh, and D. J. Pine, "Entropically driven surface phase-separation in binary colloid mixtures," Phys. Rev. Lett. 72, 582 (1994)

${ }^{17}$ K. Bayliss, J. S. van Duijneveldt, M. A. Faers, and A. W. P. Vermeer, "Comparing colloidal phase separation induced by linear polymer and by microgel particles," Soft Matter 7, 10345 (2011).

${ }^{18}$ M. Dijkstra, R. van Roij, and R. Evans, "Phase diagram of highly asymmetric binary hard-sphere mixtures," Phys. Rev. E 59, 5744 (1999).

${ }^{19}$ M. Sikorski, A. R. Sandy, and S. Narayanan, "Depletion-induced structure and dynamics in bimodal colloidal suspensions," Phys. Rev. Lett. 106, 188301 (2011)

${ }^{20}$ A. A. Louis, R. Finken, and J. P. Hansen, "Crystallization and phase separation in nonadditive binary hard-sphere mixtures," Phys. Rev. E 61, 1028 (2000).

${ }^{21} \mathrm{P}$. Germain and S. Amokrane, "Equilibrium route to colloidal gelation: Mixtures of hard-sphere-like colloids," Phys. Rev. Lett. 102, 058301 (2009).

${ }^{22} \mathrm{~A}$. Imhof and J. K. G. Dhont, "Experimental phas-diagram of a binary colloidal hard-sphere mixture with large-size ratio," Phys. Rev. Lett. 75, 1662 (1995).

${ }^{23}$ P. J. Lu, E. Zaccarelli, F. Ciulla, A. B. Schofield, F. Sciortino, and D. A. Weitz, "Gelation of particles with short-range attraction," Nature 453, 499 (2008).
${ }^{24}$ S. Manley, H. M. Wyss, K. Miyazaki, J. C. Conrad, V. Trappe, L. J. Kaufman, D. R. Reichman, and D. A. Weitz, "Glasslike arrest in spinodal decomposition as a route to colloidal gelation," Phys. Rev. Lett. 95, 238302 (2005)

${ }^{25} \mathrm{P}$. Germain and S. Amokrane, "Gelation and phase coexistence in colloidal suspensions with short-range forces: Generic behavior versus specificity," Phys. Rev. E 81, 011407 (2010).

${ }^{26} \mathrm{P}$. Germain, "Effect of residual attractive interactions in size asymmetric colloidal mixtures: Theoretical analysis and predictions," J. Chem. Phys. 133, 044905 (2010).

${ }^{27}$ A. I. Campbell, V. J. Anderson, J. S. van Duijneveldt, and P. Bartlett, "Dynamical arrest in attractive colloids: The effect of long-range repulsion," Phys. Rev. Lett. 94, 208301 (2005).

${ }^{28}$ F. Sciortino, S. Mossa, E. Zaccarelli, and P. Tartaglia, "Equilibrium cluster phases and low-density arrested disordered states: The role of shortrange attraction and long-range repulsion," Phys. Rev. Lett. 93, 055701 (2004).

${ }^{29}$ A. Stradner, H. Sedgwick, F. Cardinaux, W. C. K. Poon, S. U. Egelhaaf, and P. Schurtenberger, "Equilibrium cluster formation in concentrated protein solutions and colloids," Nature 432, 492 (2004).

${ }^{30}$ M. Pelaez-Fernandez, J. Callejas-Fernandez, and A. Moncho-Jorda, "Effective interaction in asymmetric charged binary mixtures: The non-monotonic behaviour with the colloidal charge," Eur. Phys. J. E 35, 120 (2012).

${ }^{31}$ S. A. Egorov, "Effect of repulsive and attractive interactions on depletion forces in colloidal suspensions: A density functional theory treatment," Phys. Rev. E 70, 031402 (2004).

${ }^{32}$ M. Camargo and C. N. Likos, "Unusual features of depletion interactions in soft polymer-based colloids mixed with linear homopolymers," Phys. Rev. Lett. 104, 078301 (2010).

${ }^{33}$ G. Cinacchi, Y. Martínez-Ratón, L. Mederos, G. Navascués, A. Tani, and E. Velasco, "Large attractive depletion interactions in soft repulsive-sphere binary mixtures," J. Chem. Phys. 127, 214501 (2007).

${ }^{34}$ A. Jamnik, "Effective interaction between large colloidal particles immersed in a bidisperse suspension of short-ranged attractive colloids," J. Chem. Phys. 131, 164111 (2009)

${ }^{35}$ A. Lajovic, M. Tomsic, and A. Jamnik, "Depletion effects in a mixture of hard and attractive colloids," J. Chem. Phys. 130, 104101 (2009).

${ }^{36}$ A. A. Louis, E. Allahyarov, H. Lowen, and R. Roth, "Effective forces in colloidal mixtures: From depletion attraction to accumulation repulsion," Phys. Rev. E 65, 061407 (2002).

${ }^{37}$ C. J. Rueb and C. F. Zukoski, "Viscoelastic properties of colloidal gels," J. Rheol. 41, 197 (1997).

${ }^{38}$ S. Ramakrishnan, V. Gopalakrishnan, and C. F. Zukoski, "Clustering and mechanics in dense depletion and thermal gels," Langmuir 21, 9917 (2005)

${ }^{39}$ S. Ramakrishnan and C. F. Zukoski, "Microstructure and rheology of thermoreversible nanoparticle gels," Langmuir 22, 7833 (2006).

${ }^{40}$ C. J. Rueb and C. F. Zukoski, "Rheology of suspensions of weakly attractive particles: Approach to gelation," J. Rheol. 42, 1451 (1998).

${ }^{41}$ J. W. Jansen, C. G. Dekruif, and A. Vrij, "Attractions in sterically stabilized silica suspensions: II. Experiments on phase-separation induced by temperature variation," J. Colloid Interface Sci. 114, 481 (1986).

${ }^{42}$ A. S. Negi, C. G. Redmon, S. Ramakrishnan, and C. O. Osuji, "Viscoelasticity of a colloidal gel during dynamical arrest: Evolution through the critical gel and comparison with a soft colloidal glass," J. Rheol. 58, 1557 (2014)

${ }^{43}$ H. Guo, S. Ramakrishnan, J. L. Harden, and R. L. Leheny, "Gel formation and aging in weakly attractive nanocolloid suspensions at intermediate concentrations," J. Chem. Phys. 135, 154903 (2011).

${ }^{44}$ H. Guo, S. Ramakrishnan, J. L. Harden, and R. L. Leheny, "Connecting nanoscale motion and rheology of gel-forming colloidal suspensions," Phys. Rev. E 81, 050401 (2010).

${ }^{45}$ Q. Zhang, D. Bahadur, E. M. Dufresne, P. Grybos, P. Kmon, R. L. Leheny, P. Maj, S. Narayanan, R. Szczygiel, S. Ramakrishnan, and A. R. Sandy, "Dynamic scaling of colloidal gel formation at intermediate concentrations," Phys. Rev. Lett. 119, 178006 (2017).

${ }^{46}$ B. J. Anderson, V. Gopalakrishnan, S. Ramakrishnan, and C. F. Zukoski, "Scattering for mixtures of hard spheres: Comparison of total scattering intensities with model," Phys. Rev. E 73, 031407 (2006).

${ }^{47}$ N. W. Ashcroft and D. C. Langreth, "Structure of binary liquid mixtures. I," Phys. Rev. 156, 685 (1967).

${ }^{48}$ Resolving this ambiguity would require measurements of the partial structure factors corresponding to large-large, small-small, and large-small pair correlations in the mixtures, which unfortunately is not feasible with x-ray 
or neutron scattering due to the chemically identical composition of the two colloidal species.

${ }^{49}$ A. P. R. Eberle, N. J. Wagner, and R. Castañeda-Priego, "Dynamical arrest transition in nanoparticle dispersions with short-range attraction," Phys. Rev. Lett. 106, 105704 (2011).

${ }^{50}$ M. Sztucki, T. Narayanan, G. Belina, A. Moussaïd, F. Pignon, and H. Hoekstra, "Kinetic arrest and glass-glass transition in short-ranged attractive colloids," Phys. Rev. E 74, 051504 (2006).

${ }^{51}$ M. Carta, D. Pini, A. Parola, and L. Reatto, "A density-functional theory study of microphase formation in binary Gaussian mixtures," J. Phys.: Condens. Matter 24, 284106 (2012).

${ }^{52}$ R. L. Leheny, "XPCS: Nanoscale motion and rheology," Curr. Opin. Colloid Interface Sci. 17, 3 (2012).

${ }^{53}$ A. Madsen, R. L. Leheny, H. Guo, M. Sprung, and O. Czakkel, "Beyond simple exponential correlation functions and equilibrium dynamics in X-ray photon correlation spectroscopy," New J. Phys. 12, 055001 (2010).

${ }^{54}$ L. Cipelletti, L. Ramos, S. Manley, E. Pitard, D. A. Weitz, E. E. Pashkovski, and M. Johansson, "Universal non-diffusive slow dynamics in aging soft matter," Faraday Discuss. 123, 237 (2003).

${ }^{55}$ J.-P. Bouchaud and E. Pitard, "Anomalous dynamical light scattering in soft glassy gels,” Eur. Phys. J. E 6, 231 (2001).

${ }^{56}$ W. C. K. Poon, L. Starrs, S. P. Meeker, A. Moussaïd, R. M. L. Evans, P. N. Pusey, and M. M. Robins, "Delayed sedimentation of transient gels in colloid-polymer mixtures: Dark-field observation, rheology and dynamic light scattering studies," Faraday Discuss. 112, 143 (1999).

${ }^{57}$ A. M. Puertas, M. Fuchs, and M. E. Cates, "Mode coupling and dynamical heterogeneity in colloidal gelation: A simulation study," J. Phys. Chem. B 109, 6666 (2005).

${ }^{58}$ Y.-L. Chen and K. S. Schweizer, "Microscopic theory of gelation and elasticity in polymer-particle suspensions," J. Chem. Phys. 120, 7212 (2004).

${ }^{59}$ L. M. Wang, Y. J. Tian, R. P. Liu, and K. L. Ngai, “Anomalous component dynamics of a binary mixture of associating glass-forming liquids," J. Phys. Chem. B 115, 719 (2011).

${ }^{60}$ L. M. Wang, Y. J. Tian, R. P. Liu, and R. Richert, "Structural relaxation dynamics in binary glass-forming molecular liquids with ideal and complex mixing behavior," J. Phys. Chem. B 114, 3618 (2010).

${ }^{61}$ G. Power, J. K. Vij, and G. P. Johari, "Dielectric relaxation and crystallization of nanophase separated 1-propanol-isoamylbromide mixture," J. Chem. Phys. 127, 094507 (2007).

${ }^{62}$ N. Dorsaz, G. M. Thurston, A. Stradner, P. Schurtenberger, and G. Foffi, "Phase separation in binary eye lens protein mixtures," Soft Matter 7, 1763 (2011).

${ }^{63}$ W. Stober, A. Fink, and E. Bohn, "Controlled growth of monodisperse silica spheres in micron size range," J. Colloid Interface Sci. 26, 62 (1968).
${ }^{64}$ G. H. Bogush, M. A. Tracy, and C. F. Zukoski, "Preparation of monodisperse silica particles: Control of size and mass fraction,” J. Non-Cryst. Solids 104, 95 (1988).

${ }^{65}$ A. K. Van Helden, J. W. Jansen, and A. Vrij, "Preparation and characterization of spherical monodisperse silica dispersions in non-aqueous solvents," J. Colloid Interface Sci. 81, 354 (1981).

${ }^{66}$ D. Lumma, L. B. Lurio, M. A. Borthwick, P. Falus, and S. G. J. Mochrie, "Structure and dynamics of concentrated dispersions of polystyrene latex spheres in glycerol: Static and dynamic x-ray scattering," Phys. Rev. E 62, 8258 (2000).

${ }^{67}$ D. Lumma, L. B. Lurio, S. G. J. Mochrie, and M. Sutton, "Area detector based photon correlation in the regime of short data batches: Data reduction for dynamic x-ray scattering,” Rev. Sci. Instrum. 71, 3274 (2000).

${ }^{68}$ J. A. Anderson, C. D. Lorenz, and A. Travesset, "General purpose molecular dynamics simulations fully implemented on graphics processing units," J. Comput. Phys. 227, 5342 (2008).

${ }^{69}$ J. Glaser, T. D. Nguyen, J. A. Anderson, P. Lui, F. Spiga, J. A. Millan, D. C. Morse, and S. C. Glotzer, "Strong scaling of general-purpose molecular dynamics simulations on GPUs," Comput. Phys. Commun. 192, 97 (2015).

${ }^{70}$ J. P. Hansen and I. R. McDonald, Theory of Simple Liquids, 4th ed. (Academic Press, Oxford, 2013).

${ }^{71}$ X. S. Chen and F. Forstmann, "The demixing and gas-liquid instability of a binary Yukawa fluid," J. Chem. Phys. 97, 3696 (1992).

${ }^{72}$ T. Boublík, "Hard sphere equation of state," J. Chem. Phys. 53, 471 (1970).

${ }^{73}$ G. A. Mansoori, N. F. Carnahan, K. E. Starling, and T. W. Leland, "Equilibrium thermodynamic properties of the mixture of hard spheres," J. Chem. Phys. 54, 1523 (1971).

${ }^{74}$ J. J. Salacuse and G. Stell, "Polydisperse systems: Statistical thermodynamics, with applications to several models including hard and permeable spheres," J. Chem. Phys. 77, 3714 (1982).

${ }^{75}$ N. Dorsaz, G. M. Thurston, A. Stradner, P. Schurtenberger, and G. Foffi, "Colloidal characterization and thermodynamic stability of binary eye lens protein mixtures," J. Phys. Chem. B 113, 1693 (2009).

${ }^{76}$ J. L. Lebowitz, "Exact solution of generalized Percus-Yevick equation for a mixture of hard spheres," Phys. Rev. 133, A895 (1964).

${ }^{77}$ E. W. Grundke and D. Henderson, "Distribution functions of multicomponent fluid mixtures of hard spheres," Mol. Phys. 24, 269 (1972).

${ }^{78}$ L. L. Lee and D. Levesque, "Perturbation theory for mixtures of simple liquids," Mol. Phys. 26, 1351 (1973).

${ }^{79}$ D. Henderson and E. W. Grundke, "Analytic methods for the PercusYevick hard sphere correlation functions," Condens. MatterPhys. 12, 127 (2009). 